\title{
Study on Generalized Directional Differentiability Problems of Fuzzy Mappings
}

\author{
Yu-e Bao ${ }^{1}$, Tingting Li, Linfen Zhang \\ College of Mathematics, Inner Mongolia University for Nationalities, China
}

\begin{abstract}
This paper discusses the gH-directional differentiability of fuzzy mappings, and proposes the concept of gH-directional differentiability of fuzzy mappings. Based on the concept of gH-directional differentiability of intervalvalued mappings and its related properties, two properties of gH-directional differentiability fuzzy mappings are proposed. At the same time, the relation between gH-differentiability and gH-directional differentiability for a fuzzy mapping is discussed, and it is proved that both gH-derivative and gH-partial derivative are directional derivatives of fuzzy mappings in the direction of the coordinate axis.
\end{abstract}

Keywords. Fuzzy mappings, gH-differentiability, gH-derivative, gH-partial derivative, gH-directional differentiability

\section{Introduction}

The differentiability concept of fuzzy mappings is closely related to the difference operation (subtraction operation) for fuzzy numbers. Regarding the difference operation of fuzzy numbers, its definition is relatively complicated, and there are multiple definitions. Nonetheless, there are two kinds of difference operations for fuzzy numbers, which are used to discuss the differentiability of fuzzy mappings at present, one is the concept of H-difference introduced by Hukuhara [1]; the other is the concept of generalized H-difference supported by Stefanini[2], i.e., gH-difference concept. The differentiability concept based on H-difference is called differentiability or $\mathrm{H}$ differentiability problem; the concept of differentiability established by using $\mathrm{gH}$ difference is called generalized differentiability or gH-differentiability problem. The generalized differentiability discussed in this paper refers to the problem of $\mathrm{gH}$ differentiability.

In order to overcome some deficiencies of the concept of H-differentiability, in 2013, Barnabás Bede and Luciano Stefanini [3] first discussed the differentiability of fuzzy mappings by using the gH-difference and gave the concept of gH-differentiability. Meanwhile, they put forward a new and feasible method for studying the differentiability of fuzzy mappings and its application. Since then, many scholars have studied the gH-differentiability and application of fuzzy mappings, and obtained a series of valuable results [3-11].

${ }^{1}$ Corresponding Author: Yu-e Bao, College of Mathematics, Inner Mongolia University for Nationalities. Email:byebed@163.com. 
In the study of the differentiability (H-differentiability) of fuzzy mappings, the concepts of the directional derivative (H-directional derivative) and subgradient of fuzzy mappings are proposed by Guixiang Wang and Congxin $\mathrm{Wu}$ in reference[12] , and the concepts are applied in convex fuzzy programming. However, the generalized differentiability (gH-differentiability) problem of fuzzy mappings in the above research work only considers the differentiability problem in the direction of the coordinate axis, and does not consider the differentiability in any particular directions, i.e., the gHdirectional differentiability problem. Therefore, it is necessary to discuss the gHdirectional differentiability problem of fuzzy mappings, so that the gH-differentiability of fuzzy mappings and its application can be fully discussed and developed, and some conclusions that are convenient for practical application are obtained.

In reference [13] and [14], we discussed respectively the differentiability (Hdifferentiability) and application in fuzzy programming for fuzzy mappings and the gH-directional differentiability of interval-valued mappings and so on, in the meantime, some important conclusions are obtained. On this basis, in the first section of this article, the concepts of gH-differentiability, gH-derivative, gH-partial derivative of fuzzy mappings and the concept and related properties of gH-differentiability of interval-valued mappings are given in references $[3,10,14]$. In Section 2, the problem of gH-directional differentiability of fuzzy mappings is discussed. Using the concept of gH-directional differentiability and related properties of interval-valued mappings [14], the relevant properties of gH-directional differentiability fuzzy mappings are given; And gH-directional derivative are used to characterize gH-derivative [3], gH-partial derivative [10] and gH-gradient [10].

\section{Preliminaries}

We denote by $R$ the family of all sets of real numbers, and $\mathcal{F}$ be a set of all fuzzy numbers in $R$ (or fuzzy numbers space). So, for any $u \in \mathcal{F}$, we have that its $\alpha$-level set be bounded closed intervals and we denote its $\alpha$ - levels by $[u]^{\alpha}=\left[\underline{u}_{\alpha}, \bar{u}_{\alpha}\right]$ for all $\alpha \in[0,1]$.

For fuzzy numbers $u, v \in \mathcal{F}$ and $r \in R$, respectively, the addition and the scalar multiplication are defined as follows

$$
\begin{aligned}
& {[u+v]^{\alpha}=\left[(\underline{u+v})_{\alpha},(\overline{u+v})_{\alpha}\right]=\left[\underline{u}_{\alpha}+\underline{v}_{\alpha}, \bar{u}_{\alpha}+\bar{v}_{\alpha}\right], \alpha \in[0,1] .} \\
& {[r u]^{\alpha}=\left[(\underline{r u})_{\alpha},(\overline{r u})_{\alpha}\right]=\left[\min \left\{r \underline{u}_{\alpha}, r \bar{u}_{\alpha}\right\}, \max \left\{r \underline{u}_{\alpha}, r \bar{u}_{\alpha}\right\}\right], \alpha \in[0,1] .}
\end{aligned}
$$

Definition 2.1[3]. For fuzzy numbers $u, v \in \mathcal{F}$, the generalized Hukuhara difference ( $g H$-difference, for short) of $u$ and $v$ is the fuzzy number $w \in \mathcal{F}$, if it exists, such that $u=v+w$ or $v=u+(-1) w$. And we denote as $u \Theta_{g H} v=w$.

If $u \ominus_{g H} v$ exists, then for $\alpha \in[0,1]$, in terms of $\alpha$-levels, we have

$$
\left[u \Theta_{g H} v\right]^{\alpha}=[u]^{\alpha} \Theta_{g H}[v]^{\alpha}=\left[\min \left\{\underline{u}_{\alpha}-\underline{v}_{\alpha}, \bar{u}_{\alpha}-\bar{v}_{\alpha}\right\}, \max \left\{\underline{u}_{\alpha}-\underline{v}_{\alpha}, \bar{u}_{\alpha}-\bar{v}_{\alpha}\right\}\right] .
$$

Where, $[u]^{\alpha} \Theta_{g H}[v]^{\alpha}$ is the $g H-$ difference of two interval operands [13].

Proposition 2.1[8]. For fuzzy numbers $u, v \in \mathcal{F}$, if the $g H$-difference $u \ominus_{g H} v$ 
exists, then for $r \in R$, the $r u \ominus_{g H} r v$ exists, and

$$
r\left[u \Theta_{g H} v\right]=(r u) \Theta_{g H}(r v) .
$$

For fuzzy numbers $u, v \in \mathcal{F}$, the Hausdorff distance of $u$ and $v$ is defined by

$$
D_{H}(u, v)=\sup _{\alpha \in[0,1]} d_{H}\left([u]^{\alpha},[v]^{\alpha}\right)=\sup _{\alpha \in[0,1]} \max \left\{\left|\underline{u}_{\alpha}-\underline{v}_{\alpha}\right|,\left|\bar{u}_{\alpha}-\bar{v}_{\alpha}\right|\right\} .
$$

Where, $d_{H}\left([u]^{\alpha},[v]^{\alpha}\right)=\max \left\{\left|\underline{u}_{\alpha}-\underline{v}_{\alpha}\right|,\left|\bar{u}_{\alpha}-\bar{v}_{\alpha}\right|\right\}$ is the Hausdorff distance of two interval operands $[u]^{\alpha}$ and $[v]^{\alpha}$.

Let $R^{n}$ denote the n-dimensional Euelidean space, and $M$ be a nonempty subset of $R^{n}$.We say $F: M \rightarrow \mathcal{F}$ be a fuzzy mapping (or fuzzy-valued function). For $\alpha \in[0,1]$, we can easily obtain a family of interval-valued mappings $F_{\alpha}: M \rightarrow[R]$, and we denote as $F_{\alpha}(x)=[F(x)]^{\alpha}=\left[\underline{F}_{\alpha}(x), \bar{F}_{\alpha}(x)\right], x \in M$.

Where, (1) $[R]$ indicates the interval number space formed by all bounded closed intervals in $R$ (see the reference[13]).

(2) $\underline{F}_{\alpha}(x)$ and $\bar{F}_{\alpha}(x)$ are two real-valued functions defined on $M$, called endpoint functions.

Definition2.2[3].Let $F:(a, b) \rightarrow \mathcal{F}$ be a fuzzy mapping, $x_{0} \in(a, b)$ and $x_{0}+h \in(a, b)$.Then the $g H-$ derivative of $F$ at $x_{0}$ is defined as

$$
\lim _{h \rightarrow 0} \frac{F\left(x_{0}+h\right) \ominus_{g H} F\left(x_{0}\right)}{h}=u .
$$

If exists $u \in \mathcal{F}$, we say that $F$ is gH-differentiable, and $u$ is called the $g H-$ derivative of $F$ at $x_{0}$, which is denoted as $F^{g H}\left(x_{0}\right)=u$.

Definition2.3[10]. Let $F: M \rightarrow \mathcal{F}$ be a fuzzy mapping, and $x^{0}=\left(x_{1}^{0}, x_{2}^{0}, \cdots, x_{n}^{0}\right) \in M$. If fuzzy mapping $H_{i}\left(x_{i}\right)=F\left(x_{1}^{0}, \cdots, x_{i-1}^{0}, x_{i}^{0}, x_{i+1}^{0}, \cdots, x_{n}^{0}\right)$ is $g H-$ differentiable at $x_{i}$, then we say that the $g H$ - partial derivative of $F$ at $x^{0}$ with respect to $x_{i}$, and $H_{i}^{g H}\left(x^{0}\right)$ is called the $g H-$ partial derivative of $F$ at $x^{0}$, which is denoted as $\partial F /\left.\partial x_{i}\right|_{x=x_{0}}=H_{i}^{g H}\left(x^{0}\right)$.

We consider that $F$ is $g H$-differentiable at $x^{0}$ if all the partial derivatives exist on some neighborhood of $x^{0}$.

Definition 2.4[10]. Let $F: M \rightarrow \mathcal{F}$ be a fuzzy mapping. If $F$ is $g H$-differentiable at $x_{0}$, then the $g H$-gradient of $F$ at $x_{0}$, denoted by $\left(\partial F /\left.\partial x_{1}\right|_{x=x_{0}}, \cdots, \partial F /\left.\partial x_{n}\right|_{x=x_{0}}\right)$ is a fuzzy vector, defined by

$$
\nabla F\left(x_{0}\right)=\left(\left(\partial F /\left.\partial x_{1}\right|_{x=x_{0}}, \cdots, \partial F /\left.\partial x_{n}\right|_{x=x_{0}}\right)\right) .
$$

Definition 2.5[14]. Let $f: M \rightarrow\left([R], d_{H}\right)$ be a interval-valued function, and $x \in M$. If for $y \in R^{n}$, there exists $\delta>0$ such that $x+h y_{e} \in M\left(x-h y_{e} \in M\right)$ for any 
$h \in(0, \delta)$, and there exists $A_{+} \in[R]\left(A_{-} \in[R]\right)$ such that

$$
\lim _{h \rightarrow 0^{+}} \frac{1}{h}\left(f\left(x+h y_{e}\right) \ominus_{g H} f(x)\right)=A_{+}\left(\lim _{h \rightarrow 0^{+}} \frac{1}{h}\left(f(x) \ominus_{g H} f\left(x-h y_{e}\right)\right)=A_{-}\right) .
$$

Then $f(x)$ is (resp. left) $g H$-differentiable in the direction $y$ at $x$, and $A_{+}\left(A_{-}\right)$is called the (resp. left) $g H-$ derivative of $f(x)$ in the direction $y$ at $x$, which is denoted as $f_{+}^{g H}(x, y)=A_{+}\left(f_{-}^{g H}(x, y)=A_{-}\right)$.

If $f_{+}^{g H}(x, y)=f_{-}^{g H}(x, y)$, we say that $f(x)$ is $g H$-differentiable in the direction $y$ at $x$, and denoted as $f^{g H}(x, y)=f_{+}^{g H}(x, y)=f_{-}^{g H}(x, y)$, where $f^{g H}(x, y)$ is called the $g H$-derivative of $f(x)$ in the direction $y$ at $x$.

Theorem2.1[13]. Let $F: M \rightarrow[R]$ be an interval-valued mapping, and denoted as $F(x)=[\underline{F}(x), \bar{F}(x)]$. If $\underline{F}(x)$ and $\bar{F}(x)$ are $g H$-differentiable in the direction $y$ at $x_{0}$, then $F(x)$ is $g H$-differentiable in the direction $y$ at $x_{0}$, and

$$
F^{g H}\left(x_{0}, y\right)=\left[\min \left\{\underline{F}\left(x_{0}, y\right), \bar{F}\left(x_{0}, y\right)\right\}, \max \left\{\underline{F}\left(x_{0}, y\right), \bar{F}\left(x_{0}, y\right)\right\}\right] .
$$

\section{3. gH-Directional differentiability of fuzzy mapping}

Definition 3.1. Let $F: M \rightarrow \mathcal{F}$ be a fuzzy mapping, and $x \in M$. If for $y \in R^{n}$, there exists $\delta>0$ such that $x+h y_{e} \in M\left(x-h y_{e} \in M\right)$ for $h \in(0, \delta)$. And $g H$-difference

$$
F\left(x+h y_{e}\right) \ominus_{g H} F(x)\left(F(x) \ominus_{g H} F\left(x-h y_{e}\right)\right)
$$

exists, and there exists $u^{+} \in \mathcal{F}\left(u^{-} \in \mathcal{F}\right)$ such that

$$
\lim _{h \rightarrow 0^{+}} \frac{F\left(x+h y_{e}\right) \ominus_{g H} F(x)}{h}=u^{+}\left(\lim _{h \rightarrow 0^{+}} \frac{F(x) \ominus_{g H} F\left(x-h y_{e}\right)}{h}=u^{-}\right) .
$$

Then the right (resp. left) gH-derivative of $F$ in the direction $y$ at $x$ exists, which is denoted as $u^{+}\left(u^{-}\right)$, and $F_{+}^{g H}(x, y)=u^{+}\left(F_{-}^{g H}(x, y)=u^{-}\right)$.

If $F_{+}^{g H}(x, y)=F_{-}^{g H}(x, y)$, we say that $F$ is $g H-$ derivable in the direction $y$ at $x$, and denoted as

$$
F^{g H}(x, y)=F_{+}^{g H}(x, y)=F_{-}^{g H}(x, y),
$$

Where $F^{g H}(x, y)$ is called the $g H$-derivative of $F$ in the direction $y$ at $x$.

Theorem 3.1. Let $F: M \rightarrow \mathcal{F}$ be a fuzzy mapping. If $F$ is $g H$-derivable in the direction $y \in R^{n}$ at $x \in M$, then for any $\alpha \in[0,1], F_{\alpha}: M \rightarrow[R]$ is an interval-valued mapping, which is $g H$-derivable in the direction $y$ at $x$, and

$$
F_{\alpha}^{g H}(x, y)=\left[F^{g H}(x, y)\right]^{\alpha} .
$$

Proof. Let $F: M \rightarrow \mathcal{F}$ be a fuzzy mapping, which is $g H$-derivable in the direction 
$y$ at $x \in M$, then there exists $\delta>0$ such that

$$
x+h y_{e} \in M\left(x-h y_{e} \in M\right) \text { for any } h \in(0, \delta),
$$

and $g H$ - difference

$$
F\left(x+h y_{e}\right) \ominus_{g H} F(x) \text { and }\left(F(x) \ominus_{g H} F\left(x-h y_{e}\right)\right)
$$

exist, and there exists $F^{g H}(x, y) \in \mathcal{F}$ such that

$$
\lim _{h \rightarrow 0^{+}} \frac{F\left(x+h y_{e}\right) \ominus_{g H} F(x)}{h}=\lim _{h \rightarrow 0^{+}} \frac{F(x) \ominus_{g H} F\left(x-h y_{e}\right)}{h}=F^{g H}(x, y) .
$$

That is, there exists $\delta^{\prime}>0$ such that

$$
D_{H}\left(\frac{F\left(x+h y_{e}\right) \Theta_{g H} F(x)}{h}, F^{g H}(x, y)\right)=D_{H}\left(\frac{F(x) \Theta_{g H} F\left(x-h y_{e}\right)}{h}, F^{g H}(x, y)\right)<\varepsilon
$$

for any $0<h<\delta^{\prime}$. From the Definition of $D_{H}$ and Proposition 2.1, we have

$$
d_{H}\left(\frac{1}{h}\left(\left[F\left(x+h y_{e}\right)\right]^{\alpha} \Theta_{g H}[F(x)]^{\alpha},\left[F^{g H}(x, y)\right]^{\alpha}\right)\right)=d_{H}\left(\frac{1}{h}\left([F(x)]^{\alpha} \Theta_{g H}\left[F\left(x-h y_{e}\right)\right]^{\alpha}\right)\right)<\varepsilon
$$

for any $\alpha \in[0,1]$. So,

$$
\lim _{h \rightarrow 0^{+}} \frac{\left[F\left(x+h y_{e}\right)\right]^{\alpha} \Theta_{g H}[F(x)]^{\alpha}}{h}=\lim _{h \rightarrow 0^{+}} \frac{[F(x)]^{\alpha} \Theta_{g H}\left[F\left(x-h y_{e}\right)\right]^{\alpha}}{h}=\left[F^{g H}(x, y)\right]^{\alpha} \text {. }
$$

Hence, from the Definition 2.5, $F_{\alpha}(x)=[F(x)]^{\alpha}$ is a interval-valued mapping, which is $g H$ - differentiable in the direction $y$ at $x$, and

$$
F_{\alpha}^{g H}(x, y)=\left[F^{g H}(x, y)\right]^{\alpha} \text { for any } \alpha \in[0,1] .
$$

Theorem 3.2. Let $F: M \rightarrow \mathcal{F}$ be a fuzzy mapping, which is $g H$-differentiable in the direction $y$ at $x$. If $F_{\alpha}(x)=\left[\underline{F}_{\alpha}(x), \bar{F}_{\alpha}(x)\right]$ for any $\alpha \in[0,1]$ is an intervalvalued mapping, whose two endpoint functions $\underline{F}_{\alpha}(x)$ and $\bar{F}_{\alpha}(x)$ are differentiable in the direction $y$ at $x$, then

$$
\left[F^{g H}(x, y)\right]^{\alpha}=\left[\min \left\{\underline{F}_{\alpha}(x, y), \bar{F}_{\alpha}(x, y)\right\}, \max \left\{\underline{F}_{\alpha}(x, y), \bar{F}_{\alpha}(x, y)\right\}\right] .
$$

Where $\underline{F}_{\alpha}(x, y)$ and $\bar{F}_{\alpha}(x, y)$ is the directional derivative of real-valued functions $\underline{F}_{\alpha}(x)$ and $\bar{F}_{\alpha}(x)$ in the direction $y$ at $x$ respectively.

Proof. Let $F: M \rightarrow \mathcal{F}$ is $g H$-differentiable in the direction $y$ at $x$, then from Theorem 3.1, $F_{\alpha}(x)=\left[\underline{F}_{\alpha}(x), \bar{F}_{\alpha}(x)\right]$ is an interval-valued mapping, which is $g H$-differentiable in the direction $y$ at $x$, and

$$
\left[F^{g H}(x, y)\right]^{\alpha}=F_{\alpha}^{g H}(x, y) .
$$

Moreover, for $\alpha \in[0,1], \underline{F}_{\alpha}(x)$ and $\bar{F}_{\alpha}(x)$ are $g H$-differentiable in the direction $y$ at $x$, then by Theorem 2.1, we have

$$
F_{\alpha}^{g H}(x, y)=\left[\min \left\{\underline{F}_{\alpha}(x, y), \bar{F}_{\alpha}(x, y)\right\}, \max \left\{\underline{F}_{\alpha}(x, y), \bar{F}_{\alpha}(x, y)\right\}\right] .
$$


Therefore, for $\alpha \in[0,1]$, from (1) and (2), we easily obtain

$$
\left[F^{g H}(x, y)\right]^{\alpha}=\left[\min \left\{\underline{F}_{\alpha}(x, y), \bar{F}_{\alpha}(x, y)\right\}, \max \left\{\underline{F}_{\alpha}(x, y), \bar{F}_{\alpha}(x, y)\right\}\right]
$$

Theorem 3.3. Let $F:(a, b) \rightarrow \mathcal{F}$ be a fuzzy mapping, then $F$ is $g H$-differentiable in the direction $y=1$ at $x$ if and only if $F$ is $g H$-differentiable at $x$, and

$$
F^{g H}(x)=F^{g H}(x, 1) \text {. }
$$

Proof. Necessity. If $F(x)$ is $g H$-differentiable in the direction $y=1$ at $x$, then there exists $\delta>0$ such that $x-h, x+h \in(a, b)$ and for any $h \in(0, \delta), g H-$ difference

$$
F(x+h) \ominus_{g H} F(x) \text { and } F(x) \ominus_{g H} F(x-h)
$$

exist and there exists $F^{g H}(x, 1) \in \mathcal{F}$ such that

$$
\lim _{h \rightarrow 0^{+}} \frac{F(x+h) \ominus_{g H} F(x)}{h}=\lim _{h \rightarrow 0^{+}} \frac{F(x) \ominus_{g H} F(x-h)}{h}=F^{g H}(x, 1) .
$$

Moreover, we easily imply that

$$
\begin{aligned}
& -\left[F(x-h) \Theta_{g H} F(x)\right]^{\alpha}=-\left([F(x-h)]^{\alpha} \Theta_{g H}[F(x)]^{\alpha}\right) \\
& =\left[F_{g H}(x)\right]^{\alpha} \Theta_{g H}[F(x-h)]^{\alpha}
\end{aligned}
$$

for $\alpha \in[0,1]$. So,

$$
\begin{aligned}
& \lim _{h \rightarrow 0^{-}} D_{H}\left(\frac{F(x+h) \Theta_{g H} F(x)}{h}, F^{g H}(x, 1)\right) \\
= & \lim _{h \rightarrow 0^{+}} D_{H}\left(\frac{F(x-h) \Theta_{g H} F(x)}{-h}, F^{g H}(x, 1)\right) \\
= & \lim _{h \rightarrow 0^{+}} \sup _{\alpha \in[0,1]} d_{H}\left(\left[\frac{F(x-h) \Theta_{g H} F(x)}{-h}\right]^{\alpha},\left[F^{g H}(x, 1)\right]^{\alpha}\right) \\
= & \lim _{h \rightarrow 0^{+}} \sup _{\alpha \in[0,1]} d_{H}\left(\left[\frac{F(x) \Theta_{g H} F(x-h)}{h}\right]^{\alpha},\left[F^{g H}(x, 1)\right]^{\alpha}\right) \\
= & \lim _{h \rightarrow 0^{+}} D_{H}\left(\frac{F(x) \Theta_{g H} F(x-h)}{h}, F^{g H}(x, 1)\right)=0 .
\end{aligned}
$$

Therefore,

$$
\lim _{h \rightarrow 0^{-}} \frac{F(x+h) \ominus_{g H} F(x)}{h}=\lim _{h \rightarrow 0^{+}} \frac{F(x+h) \ominus_{g H} F(x)}{h}=F^{g H}(x, 1) .
$$

Hence $F$ is $g H$-derivable at $x$, and

$$
F^{g H}(x)=F^{g H}(x, 1) \text {. }
$$

Sufficiency. If $F$ is $g H-$ differentiable at $x$, i.e.,

$$
\lim _{h \rightarrow 0} \frac{F(x+h) \ominus_{g H} F(x)}{h}=F^{g H}(x) \in \mathcal{F},
$$


Then there exists $\delta>0$ such that $x+h \in(a, b)$ and for any $0<|h|<\delta, g H-$ difference $F(x+h) \ominus_{g H} F(x)$ exists and

$$
\lim _{h \rightarrow 0^{+}} \frac{F(x+h) \ominus_{g H} F(x)}{h}=F^{g H}(x)=F_{+}^{g H}(x, 1) .
$$

Moreover, $x-h \in(a, b)$ and $g H-$ difference $F(x+h) \ominus_{g H} F(x)$ exists for any $0<h<\delta$. So, from Proposition 2.1 and the operation properties of fuzzy number, we can deduce $F(x) \ominus_{g H} F(x-h)=-\left(F(x+h) \ominus_{g H} F(x)\right)$ and

$$
\begin{aligned}
& \lim _{h \rightarrow 0^{+}} D_{H}\left(\frac{F(x) \ominus_{g H} F(x-h)}{h}, F^{g H}(x)\right)=\lim _{h \rightarrow 0^{+}} D_{H}\left(\frac{F(x-h) \ominus_{g H} F(x)}{-h}, F^{g H}(x)\right) \\
& =\lim _{h \rightarrow 0^{-}} D_{H}\left(\frac{F(x+h) \ominus_{g H} F(x)}{h}, F^{g H}(x)\right)=0
\end{aligned}
$$

Therefore,

$$
\lim _{h \rightarrow 0^{+}} \frac{F(x) \ominus_{g H} F(x-h)}{h}=F^{g H}(x)=F_{-}^{g H}(x, 1) .
$$

Theorem 3.4. Let $F: M \rightarrow \mathcal{F}$ be a fuzzy mapping, and $x^{0}=\left(x_{1}^{0}, x_{2}^{0}, \cdots, x_{n}^{0}\right) \in M$. If $F(x)$ is $g H$-differentiable in the direction $e_{i}(i=1,2, \cdots, n)$ at $x^{0}$, then the partial $g H$-derivative of $F(x)$ exist at $x^{0}$ with respect to $x_{i}$, and

$$
\partial F^{g H} /\left.\partial x_{i}\right|_{x=x^{0}}=F^{g H}\left(x^{0}, e_{i}\right) .
$$

Proof. Let $H_{i}\left(x_{i}\right)=F\left(x_{1}^{0}, \cdots, x_{i-1}^{0}, x_{i}^{0}, x_{i+1}^{0}, \cdots, x_{n}^{0}\right)$, then

$$
\begin{aligned}
& \frac{H_{i}\left(x_{i}^{0}+h\right) \ominus_{g H} H_{i}\left(x_{i}^{0}\right)}{h}=\frac{F\left(x^{0}+h e_{i}\right) \ominus_{g H} F\left(x^{0}\right)}{h}, \\
& \frac{H_{i}\left(x_{i}^{0}\right) \ominus_{g H} H_{i}\left(x_{i}^{0}-h\right)}{h}=\frac{F\left(x^{0}\right) \ominus_{g H} F\left(x^{0}-h e_{i}\right)}{h} .
\end{aligned}
$$

If $F(x)$ is $g H$-differentiable in the direction $e_{i}$ at $x^{0}$, i.e.,

$$
\lim _{h \rightarrow 0^{+}} \frac{F\left(x^{0}+h e_{i}\right)-F\left(x^{0}\right)}{h}=\lim _{h \rightarrow 0^{+}} \frac{F\left(x^{0}\right)-F\left(x^{0}-h e_{i}\right)}{h}=F^{g H}\left(x^{0}, e_{i}\right) .
$$

Therefore,

$$
\lim _{h \rightarrow 0^{+}} \frac{H_{i}\left(x_{i}^{0}+h\right) \ominus_{g H} H_{i}\left(x_{i}^{0}\right)}{h}=\lim _{h \rightarrow 0^{-}} \frac{H_{i}\left(x_{i}^{0}\right) \ominus_{g H} H_{i}\left(x_{i}^{0}-h\right)}{h}=F_{i}^{g H}\left(x^{0}, e_{i}\right) .
$$

Hence $H_{i}^{g H}\left(x_{i}^{0}, 1\right)=F^{g H}\left(x^{0}, e_{i}\right)$.

From Definition 2.2, we have $H_{i}\left(x_{i}\right)$ is $g H-$ differentiable at $x_{i}^{0}$. So by Definition 2.3, the partial $g H$-derivative of $F(x)$ at $x^{0}$ respect to $x_{i}$, and denoted as

$$
\partial F^{g H} /\left.\partial x_{i}\right|_{x=x^{0}}=F^{g H}\left(x^{0}, e_{i}\right) .
$$


Corollary 3.1. Let $F: M \rightarrow \mathcal{F}$ be a fuzzy mapping, and $x^{0}=\left(x_{1}^{0}, x_{2}^{0}, \cdots, x_{n}^{0}\right) \in M$. If all the partial $g H$-derivatives exist in the direction $e_{i}$ at $x^{0}$ on some neighborhood of $x^{0}$, then $F$ is $g H$-differentiable at $x^{0}$, and its the gradient

$$
\nabla F^{g H}\left(x^{0}\right)=\left\{F^{g H}\left(x^{0}, e_{1}\right), \cdots, F^{g H}\left(x^{0}, e_{i}\right), \cdots, F^{g H}\left(x^{0}, e_{n}\right)\right\} .
$$

Proof. If $F^{g H}\left(x^{0}, e_{i}\right)(i=1,2, \cdots n)$ exist on some neighborhood of $x^{0}$, then from Theorem 3.4, we have all the partial derivatives $\partial F^{g H} /\left.\partial x_{i}\right|_{x=x^{0}}(i=1,2, \cdots n)$ exist on some neighborhood of $x^{0}$, and

$$
\partial F^{g H} /\left.\partial x_{i}\right|_{x=x^{0}}=F^{g H}\left(x^{0}, e_{i}\right)(i=1,2, \cdots n) .
$$

Therefore, from Definition 2.3, $F$ is $g H$-differentiable at $x^{0}$, and the gradient is denoted as

$$
\nabla F^{g H}\left(x^{0}\right)=\left\{F^{g H}\left(x^{0}, e_{1}\right), \cdots, F^{g H}\left(x^{0}, e_{i}\right), \cdots, F^{g H}\left(x^{0}, e_{n}\right)\right\} .
$$

\section{Conclusions}

The differentiability of fuzzy mappings is an important concept in fuzzy analysis and plays a very important role in fuzzy optimization theory. In this paper, we discuss the gH-directional differentiability of fuzzy mappings. The concept of gH-directional derivative is proposed, and gH-derivative and gH-partial derivative are described by using gH-directional derivative. These conclusions lay a solid foundation for further discussion on the gH-differentiability, gH-subdifferentiability of fuzzy mappings and their application in fuzzy programming. We will give some examples of corresponding model implementation in the next research work.

\section{Acknowledgments}

Inner Mongolia Natural Science Foundation of China (2018MS01010).

\section{References}

[1] M.Puri, D.Ralescu, Differentials of fuzzy functions, J. Math. Anal. Appl., 1983 (91),552-558.

[2] L.Stefanini, A generalization of Hukuhara difference and division for interval and fuzzy arithmetic, Fuzzy Sets Syst., 2010 (161),1564-1584.

[3] B.Bede, L.Stefanini, Generalized differentiability of fuzzy-valued functions, Fuzzy Sets Syst., 2013 (230),119-141.

[4] T.Allahviranloo, Z.Gouyandeh, A.Armand, A.Hasanoglu, On fuzzy solutions for heat equation based on generalized Hukuhara differentiability, Fuzzy Sets Syst., 2015 (265),1-23.

[5] B. Barnabás, G. Gal, Generalizations of the differentiability of fuzzy-number-valued functions with applications to fuzzy differential equations, Fuzzy Sets Syst., 2015 (151), 581-599.

[6] L.C. De Barros, F. Santo Pedro, Fuzzy differential equations with interactive derivative, Fuzzy Sets Syst., 2017 (309),64-80.

[7] G.Zengtai, H.Yang, Ill-posed fuzzy initial-boundary value problems based on generalized differentiability and regularization, Fuzzy Sets Syst., 2016 (295),99-113. 
[8] Y.Chalco-Canoa, R.Rodríguez-Lópezb, M.D.Jiménez-Gameroc, Characterizations of generalized differentiable fuzzy functions, Fuzzy Sets Syst., 2016 (259),37-56.

[9] H.Shexiang, G.Zengtai, L.Hongxia, Generalized differentiability for n-dimensional fuzzy-number-valued functions and fuzzy optimization, Inf. Sci., 2016 (374),151-163.

[10] R.Osuna-Gómez, Y.Chalco-Canob, B.Hernández-Jiménezc, I.Aguirre-Cipe, Optimality conditions for fuzzy constrained programming problems, Fuzzy Sets Syst., 2019 (362), 35-54.

[11] Luciana T. Gomes,Laécio C. Barros,A note on the generalized difference and the generalized differentiability, Fuzzy Sets Syst., 2015 (280),142-145.

[12] B.Yu-e, B. Eer-dun, Optimality conditions for fuzzy programming problems with differentiable fuzzy objective mappings, Journal of Information Science and Engineering, 2019(35), 1311-1327.

[13] B.Yu-e, Z.Bo, B. Eer-dun, Directional differentiability of interval-valued functions, J.Math. Computer Sci., 2016 (16),507-515.

[14] Y. R. Syau, Invex and generalized convex fuzzy mappings, Fuzzy Sets Syst., 2000 (115), 455-461. 